\title{
Directivity of an Antenna Embedded Inside a Fabry-Perot Cavity: Analysis and Design
}

\author{
H. Boutayeb ${ }^{1}$, K. Mahdjoubi ${ }^{2}$, A.-C. Tarot $^{2}$ and T.A. Denidni ${ }^{1}$ \\ ${ }^{1}$ INRS-EMT, University of Québec, 800 rue de la Gauchetiere, Montréal Québec H5A 1K6, \\ Canada.boutayeb@inrs-emt.uquebec.ca. Fax: (514)-875-0344 \\ ${ }^{2}$ IETR (Institut d' Électronique et de Télécommunications de Rennes), UMR CNRS 6164, \\ Avenue du Général Leclerc, 35042 Rennes, France.
}

Abstract - The enhancement of directivity of a monopole located in a Fabry-Perot type cavity is studied. The analysis is based on the response of the cavity excited from its inside by electromagnetic waves. To validate the proposed antenna, an experimental prototype was designed, fabricated and measured.

Key words: Periodic structures; Fabry-Perot cavity; Directive antennas 


\section{Introduction}

Directive and low-cost antennas composed of a single feed present an attractive solution for several wireless communication systems, such as high-speed wireless LANs, satellite systems and point-to-point links. Their single-feed system allows to increase the gain with low complexity compared to feeding networks used in conventional antenna arrays. In this paper, a directive antenna composed of a monopole embedded inside a Fabry-Perot cavity is proposed.

Recently, several authors have developed techniques to obtain high directive antennas by embedding a dipole inside a Fabry-Perot cavity [1-3]. In [1], a relation between the half power beamwidth and the quality factor of the cavity (at the resonant frequency of the cavity) has been proposed and validated numerically, whereas in [2], it has been demonstrated that this directivity improvement has an analogy with past research in optical physics. In [3], a study on the input impedance of the antenna has been presented. In $[4,5]$, an antenna based on a Electromagnetic Band Gap (EBG) material with a defect has been proposed. The antenna consists of a Fabry-Perot cavity between a patch antenna and the EBG material.

Another way for producing a high-gain antenna has been introduced in the 50's [6]. This technique uses a Partially Reflecting Surface (PRS) to introduce leaky waves and beamforming effects when placed in front of a grounded waveguide aperture. A ray theory has been proposed, showing that the directivity of the antenna increases when the reflectivity of the PRS increases. This kind of antenna has been revisited recently [7], where the PRS has been optimized to enlarge the antenna bandwidth.

To our knowledge, the directivity at different frequencies of antennas based on a Fabry-Perot cavity has not been sufficiently studied. Indeed, the directivity (or the beamwidth) is often calculated at the resonant frequency of the cavity. However, as it is shown in this paper, the maximum of the directivity (or minimum of the beamwidth) is obtained at another frequency. In this contribution, a ray method is applied to predict the focusing characteristics of the proposed antenna. To demonstrate the proposed approach, an antenna prototype was build and measured, and the experimental results show a good agreement with the predicted ones.

\section{Analysis}

The analysis procedure for the proposed antenna is given in the following sections. In Section 2.1, the structure is characterized using the frequency and angular responses of the Fabry-Perot 
cavity to a plane wave excitation, which is in the center of the cavity. This frequency and angular function is called T. Usually, in the Fabry-Perot interferometer, the source is outside the FabryPerot cavity [8], whereas here the source is placed inside the cavity, which necessitates new formulas for the characterization. Using the calculated function $T$, in Sections 2.2 and 2.3, the relationships of the bandwidths at the half-power and at an arbitrary power ratio are developed and presented. In Section 2.4, these relations are then used to calculate new results for the half power beamwidth of the radiation pattern of the Fabry-Perot-antenna, and the main results are finally applied in Section 2.5 for the antenna design.

\subsection{Characterization}

Figure 1(a) shows the geometry of the proposed structure. This structure consists of two metallic wire rows located on each side of a point source, where a TM-wave excitation is considered. The wires are spaced periodically with the period $P$, the distance between the two rows is $D$ and the diameter of the wires is $a$. In order to characterize the structure in terms of frequency and radiation pattern, the rays going at the direction $\theta$ are considered (Fig. 1(b)). The rows of wires act as partially reflecting surfaces to these rays, with the complex reflection and transmission coefficients $r$ and $t$. An infinite number of rays exit from the cavity at the direction $\theta$. The amplitude of the direct transmitted ray $\left(t_{0}\right)$, using an arbitrary reference of phase, is equal to $t$. The amplitude of the once-reflected ray $\left(t_{1}\right)$ can be written as

$$
t_{1}=\operatorname{tr} e^{-\frac{j k D}{2 \cos (\theta)}+j k D / 2 \tan (\theta) \sin (\theta)}=\operatorname{tr} e^{-j k D \cos (\theta)}
$$

where $k=\frac{2 \pi f}{c}$ is the free space wave number, $f$ is the frequency and $c$ is the velocity of wave propagation in free space. In Eq. (1), $-\frac{k D}{2 \cos (\theta)}$ represents the phase variation during displacement and $k D / 2 \tan (\theta) \sin (\theta)$ is the path difference between ray 1 and ray 0 . Thus, the amplitude of the nth-reflected transmitted ray $\left(t_{n}\right)$ is written

$$
t_{n}=t r^{n} e^{-j n k D \cos (\theta)}
$$

The total transmitted amplitude is then written as

$$
T=\sum_{n=0}^{\infty} t_{n}=t \sum_{n=0}^{\infty} r^{n} e^{-j n k D \cos (\theta)}=\frac{t}{1-r e^{-j k D \cos (\theta)}}
$$


Using the energy conservation relation $|t|^{2}=1-|r|^{2}$ [8] and after simplifications, the squared modulus of $T$ can be written

$$
|T|^{2}=\frac{1-|r|^{2}}{1+|r|^{2}-2|r| \cos \left(k D \cos (\theta)-\varphi_{r}\right)}
$$

where $\varphi_{r}$ is the phase of $r$.

In order to obtain simple analytical results, the reflection and transmission coefficients $r$ and $t$ are considered constant (i.e. independent of the frequency and angle). This approximation is usually considered valid in the frequency band of interest $[6,7]$.

When the resonance condition $k D \cos (\theta)=\varphi_{r}$ is verified, $|T|^{2}$ achieves its maximum value $|T|_{\max }^{2}$

$$
|T|_{\max }^{2}=\frac{1-|r|^{2}}{1+|r|^{2}-2|r|}=\frac{1+|r|}{1-|r|}
$$

For instance, we consider $|r|=0.824, \varphi_{r}=2.52 \mathrm{rad}$, and $D=40 \mathrm{~mm}$. In Fig. 2, $|T|$ is plotted versus frequency at $\theta=0^{\circ}$ in the first abscise, and versus $\cos (\theta)$ at the resonant frequency $f_{0}=3 \mathrm{GHz}$ in the second abscise. The bandwidth of $|T|$ versus frequency at its half squared maximum amplitude is defined as $1 / Q$, where $Q$ is the quality factor of the cavity [1]. Figure 3 shows $|T|$ versus angle at different frequencies. These radiation patterns exhibit directive beams at the normal directions $\left(\theta=0^{\circ}\right.$ and $\left.\theta=180^{\circ}\right)$ for frequencies lower than $f_{0}$. For frequencies greater than $f_{0}$, lobs appear on each side of the normal axis. $\Delta \theta_{3 d B}$ is defined as the half power beamwidth of the main lobs at the normal directions (see Fig. 3). In the next sections, the quality factor $Q$ is first written as function of the reflection coefficient $r$, and then, the interrelation between the half power beamwidth and the quality factor $Q$ is developed.

\subsection{Half power bandwidth}

To find the expression of $1 / Q$ as a function of the reflection coefficient $r$, the following equation is resolved

$$
\frac{1-|r|^{2}}{1+|r|^{2}-2|r| \cos \left(\psi_{ \pm}-\varphi_{r}\right)}=\frac{1}{2}|T|_{\max }^{2}=\frac{1}{2} \frac{1+|r|}{1-|r|}
$$


where $\psi=k D \cos (\theta)$, and $\psi_{+}$and $\psi_{-}$are the values of $\psi$ corresponding to the half of the maximum of $|T|^{2}$. Then, resolving Eq. (6) gives

$$
\psi_{ \pm}=\varphi_{r} \pm \arccos \left(1-\frac{1}{2} \frac{(1-|r|)^{2}}{|r|}\right)
$$

$1 / Q$ can be expressed as

$$
\begin{aligned}
\frac{1}{\mathrm{Q}} & =2 \frac{\psi_{+}-\psi_{-}}{\psi_{+}+\psi_{-}}=2 \frac{f_{+}-f_{-}}{f_{+}+f_{-}} \\
& =\frac{2}{\varphi_{r}} \arccos \left(1-\frac{1}{2} \frac{(1-|r|)^{2}}{|r|}\right) \approx 2 \frac{1-|r|}{\varphi_{r} \sqrt{|r|}}
\end{aligned}
$$

The approximate relation is obtained by considering $|r|$ close to 1 , which is the condition to obtain high directivity $[6,7]$.

\subsection{Bandwidth for an arbitrary power ratio $x$}

The half power bandwidth $1 / Q$ is obtained for $|T|_{\max }^{2} / 2$. We can also define a bandwidth $1 / Q_{x}$ determined for $|T|_{\max }^{2} / x$. For this, we have to resolve the following equation :

$$
\frac{1-|r|^{2}}{1+|r|^{2}-2|r| \cos \left(\psi_{x \pm}-\varphi_{r}\right)}=\frac{1}{x} \frac{1+|r|}{1-|r|}
$$

which leads to the following expression of $1 / Q_{x}$ :

$$
\frac{1}{\mathrm{Q}_{\mathrm{x}}}=2 \frac{\psi_{x+}-\psi_{x-}}{\psi_{x+}+\psi_{x-}}=\frac{2}{\varphi_{r}} \arccos \left(1-\frac{1}{2}(x-1) \frac{(1-|r|)^{2}}{|r|}\right) \approx \sqrt{x-1} \frac{1}{Q}
$$

where $x=|T|_{\max }^{2} /|T|^{2}$. The approximate relation is obtained by considering $|r|$ close to 1 and $\boldsymbol{x}$ is such that $(x-1)(1-|r|)^{2} /|r|<<1$. Then, the approximate relation is valid for frequencies near the resonance.

\subsection{Half power beamwidth}

The bandwidth obtained for $|T|_{\max }^{2} /(2 x)$ is noted $1 / Q_{2 x}$. To resolve the interrelation between the angular beamwidth and the frequency bandwidth, a graphical method that is described in this 
section is used. Two cases are considered: the case $f \leq f_{0}$, and the case $f_{0} \leq f \leq f_{+}$. Note that $\Delta \theta_{3 d B}$ is not defined at $f>f_{+}$, because for these frequencies, the level of $|T|^{2}$ at normal is inferior to the half of $|T|_{\max }^{2}$. The link between the beamwidth $\Delta \theta_{3 d B}$ and the bandwidths $1 / Q_{x}$ and $1 / Q_{2 x}$ is shown in Fig. 4, for $f \leq f_{0}$. From this figure, the following relation can be obtained

$$
\frac{1}{\mathrm{Q}_{2 \mathrm{x}}}-\frac{1}{\mathrm{Q}_{\mathrm{x}}}=2\left(1-\cos \left(\frac{\Delta \theta_{3 d B}}{2}\right)\right)
$$

With the approximation of a small beamwidth, the relation can be written :

$$
\frac{1}{\mathrm{Q}_{2 \mathrm{x}}}-\frac{1}{\mathrm{Q}_{\mathrm{x}}} \approx\left(\frac{\Delta \theta_{3 d B}}{2}\right)^{2}
$$

Using Eq. (10), the beamwidth is then given by

$$
\Delta \theta_{3 d B, f \leq f_{0}} \approx 2 \sqrt{\frac{\sqrt{2 x-1}-\sqrt{x-1}}{Q}}
$$

Figure 5 shows the relationship between the beamwidth $\Delta \theta_{3 d B}$ and the bandwidths $1 / Q$ and $1 / Q_{x}$ when $f_{0} \leq f \leq f_{+}$. From the curve presented in Fig. 5, the following equation can be obtained

$$
\frac{1}{\mathrm{Q}_{\mathrm{x}}}-\frac{1}{\mathrm{Q}}=2\left(1-\cos \left(\frac{\Delta \theta_{3 d B}}{2}\right)\right)
$$

Then, at $f_{0} \leq f \leq f_{+}$, with the approximation of a small beamwidth, and using Eq. (10), the beamwidth can be expressed as

$$
\Delta \theta_{3 d B, f_{0} \leq f \leq f} \approx 2 \sqrt{\frac{\sqrt{x-1}+1}{Q}}
$$

Now, the link between $\Delta \theta_{3 d B}$ and $1 / Q$ is obtained for all frequencies $f \leq f_{+}$. Figure 6 exhibits the beamwidth versus frequency using Eqs. (13) and (15). From this figure, it can be seen that the minimum of $\Delta \theta_{3 d B}$ is obtained for $f \leq f_{0}$. By minimizing Eq. (13), one can see that the minimum of $\Delta \theta_{3 d B}$ is obtained for $x=1.5$, and then it can be written

$$
\Delta \theta_{3 d B \min } \approx \sqrt{\frac{2}{Q}}
$$


The frequency $f_{\Delta \theta_{3 d B} \min }$ corresponding to this value is obtained from

$$
2 \frac{f_{0}-f_{\Delta \theta_{3 d \mathrm{Bmin}}}}{f_{0}}=\frac{1}{Q_{1.5}} \approx \frac{\sqrt{0.5}}{Q}
$$

Then

$$
f_{\Delta \theta_{3 \mathrm{aBm} \text { min }}} \approx f_{0}\left(1-\frac{1}{2 \sqrt{2} Q}\right)
$$

For $|r|$ close to 1 , the quality factor $Q$ is high and then $f_{\Delta \theta_{3 \alpha B} \min }$ is close to $f_{0}$.

\subsection{Applications}

From the previous results, considering $|r|$ close to 1 , the minimum of the beamwidth $\Delta \theta_{3 d B}$ is obtained at a frequency $f_{\Delta \theta_{3 d B} \min }$, which is slightly smaller than the resonance $f_{0}$. This frequency can be written as

$$
f_{\Delta \theta_{3 \mathrm{BSm} \min }} \approx f_{0}\left(1-\frac{1}{2 \sqrt{2} Q}\right)
$$

At $f_{\Delta \theta_{3 d B} \min }$, the beamwidth $\Delta \theta_{3 d B}$ is given by

$$
\Delta \theta_{3 d B \min } \approx \sqrt{\frac{2}{Q}}
$$

At $f_{0}$, using Eq. (15) with $x=1$, the following relation is obtained

$$
\Delta \theta_{3 d B, f_{0}} \approx 2 \sqrt{\frac{1}{Q}}
$$

The quality factor $Q$ is obtained from the following equation

$$
\frac{1}{Q} \approx 2 \frac{1-|r|}{\varphi_{r} \sqrt{|r|}}
$$

The knowledge of the reflection coefficient $r$ allows then to obtain important parameters of the radiation patterns of the proposed antenna. For this, numerical simulations using the Finite Difference Time Domain (FDTD) method were carried out to obtain the reflection coefficient $r$, of an infinite grid of metallic wires with the following characteristics: $P=20 \mathrm{~mm}$ and $a=2 \mathrm{~mm}$. In Fig. 7, $|T|$ is plotted using Eq. (4) and the computed coefficient $r$, which is considered now dependent on the frequency. From this figure, the resonant frequency of the 
structure is $f_{0}=3 \mathrm{GHz}$. The modulus and phase of $r$ at $f_{0}$ are 0.824 and $2.52 \mathrm{rad}$, respectively. These values are used to calculate $1 / Q$ in Eq. (22). Then, it is possible to evaluate the frequency of maximum directivity and the beamwidth at this frequency and at the resonant frequency using Eqs.(19)-(21).

In the following section, results from Eqs.(19)-(21) are compared with experimental data.

\section{Experimental results}

To validate our approach, a prototype of the structure was fabricated and measured. A monopole is used as an excitation source. Figure 8 gives the photograph of the fabricated antenna. The wires are $50 \mathrm{~cm}$ length and $2 \mathrm{~mm}$ diameter, the number of wires in each row is 28 , the cavity is $D=40 \mathrm{~mm}$ width, the period is $P=20 \mathrm{~mm}$, the monopole is $15 \mathrm{~mm}$ length and $2 \mathrm{~mm}$ diameter. The dimensions of the ground plane are $61 \times 21 \mathrm{~cm}^{2}$.

The radiation patterns of the proposed antenna were measured in an anechoic chamber located at INRS, in Montreal. As an example, the measured radiation patterns of the antenna in the H-plane and E-plane at $2.85 \mathrm{GHz}$ are plotted in Fig. 9, showing two directive beams at the normal directions. Figure 10 exhibits the measured directivity versus frequency. From this curve, the maximum of directivity (corresponding to the minimum of beamwidth) is obtained at $2.85 \mathrm{GHz}$. From Eq. (19), it is obtained $f_{\Delta \theta_{3 A B} \min } \approx 2.846 \mathrm{GHz}$, which shows a good agreement with measured ones. The measured beamwidths are $28.8^{\circ}$ and $41.2^{\circ}$ at $2.85 \mathrm{GHz}$ and $3 \mathrm{GHz}$, respectively. The results obtained from Eqs. (20) and (21) are $30.8^{\circ}$ and $43.6^{\circ}$, these show also a good agreement with the previous experimental data.

To conclude the experimental part, note that the matching of the antenna can be done at the frequency of maximum directivity using conventional techniques (e.g., stubs on the feeding line).

\section{Conclusion}

An analysis for enhancing the directivity of an antenna based on a Fabry-Perot cavity has been presented. The frequency of maximum directivity and the beamwidth at different frequencies have been predicted with new analytical expressions. An antenna prototype has been fabricated and tested, and a good agreement has been achieved between theoretical and experimental results showing the usefulness of the proposed approach. 


\section{REFERENCES}

1. T. Akalin, J. Danglot, O. Vanbesien, and D. Lippens, A Highly Directive Dipole Antenna Embedded in a Fabry-Perot Type Cavity, IEEE Microwave Wireless Compon Lett 12 (2002), 48-50.

2. R. Biswas, E. Ozbay, B. Temelkuran, M. Bayandir, M. Sigalas, and K.-M. Ho, Exceptionally directional sources with Photonic Band-Gap crystals, Optical Society of America 18 (2001), 1684-1689.

3. H. Boutayeb, K. Mahdjoubi, and A.C. Tarot, Design of a Directive and Matched Antenna with a planar EBG structure, IEEE Antennas and Propagat Society International Symposium, AP-1 (2004), 835-838.

4. M. Thevenot, C. Cheypre, A. Reineix and B. Jecko, Directive Photonic Band-Gap Antennas, IEEE Trans Microwave Theory Tech 47 (1999), 2115-2122.

5. C. Serier, C. Cheype, R. Chantalat, M. Thevenot, T. Monediere, A. Reineix, and B. Jecko, 1D photonic bandgap resonator antenna, Microwave Opt Technol Lett 29 (2001), 312-315.

6. G.V. Trentini, Partially reflecting sheet arrays, IRE Trans Antennas Propagat 4 (1956), 666671.

7. A.P. Feresidis and J.C. Vardaxoglou, High gain planar antenna using optimized partially reflective surfaces, IEE Microw Antennas Propagat 148 (2001), 345-350.

8. E. Hecht, Optics, Addison Wesley, San Francisco, CA, 2002, 421-425. 


\section{Figure Captions:}

FIG. 1: (a) Geometry of the antenna based on a Fabry-Perot cavity

(b) Sum of the transmitted rays, outside the cavity, at the direction $\theta$.

FIG. 2: $|T|$ versus frequency (at $\theta=0^{\circ}$ ), and versus $\cos (\theta)$ (at $f_{0}$ ).

FIG. 3: Normalized $|T|$ versus $\theta$ (logarithm scale) at different frequencies.

FIG. 4: Relationship between $\Delta \theta_{3 d B}, 1 / Q_{x}$ and $1 / Q_{2 x}$ at $f \leq f_{0}$.

FIG. 5: Relationship between $\Delta \theta_{3 d B}, 1 / Q$ and $1 / Q_{x}$ at $f_{0} \leq f \leq f_{+}$.

FIG. 6: Beamwidth $\Delta \theta_{3 d B}$ versus frequency.

FIG. 7: $|T|$ at $\theta=0^{\circ}$ (computed with Matlab, using $r$, calculated with a FDTD method).

FIG. 8: Photograph of the fabricated antenna. The monopole is fed via an SMA connector.

FIG. 9: Measured radiation patterns at $2.85 \mathrm{GHz}$.

FIG. 10: Measured directivity in the H-plane versus frequency 
FIG. 1

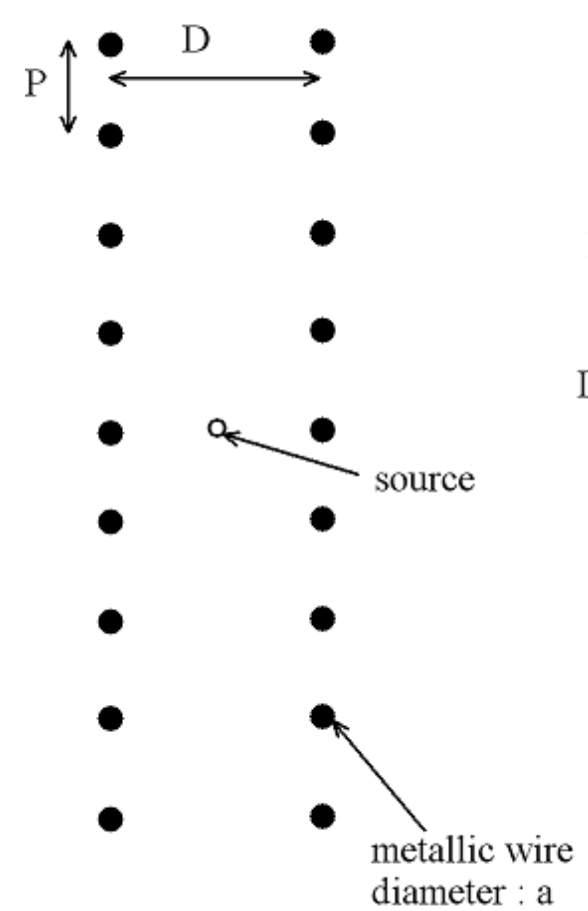

(a)

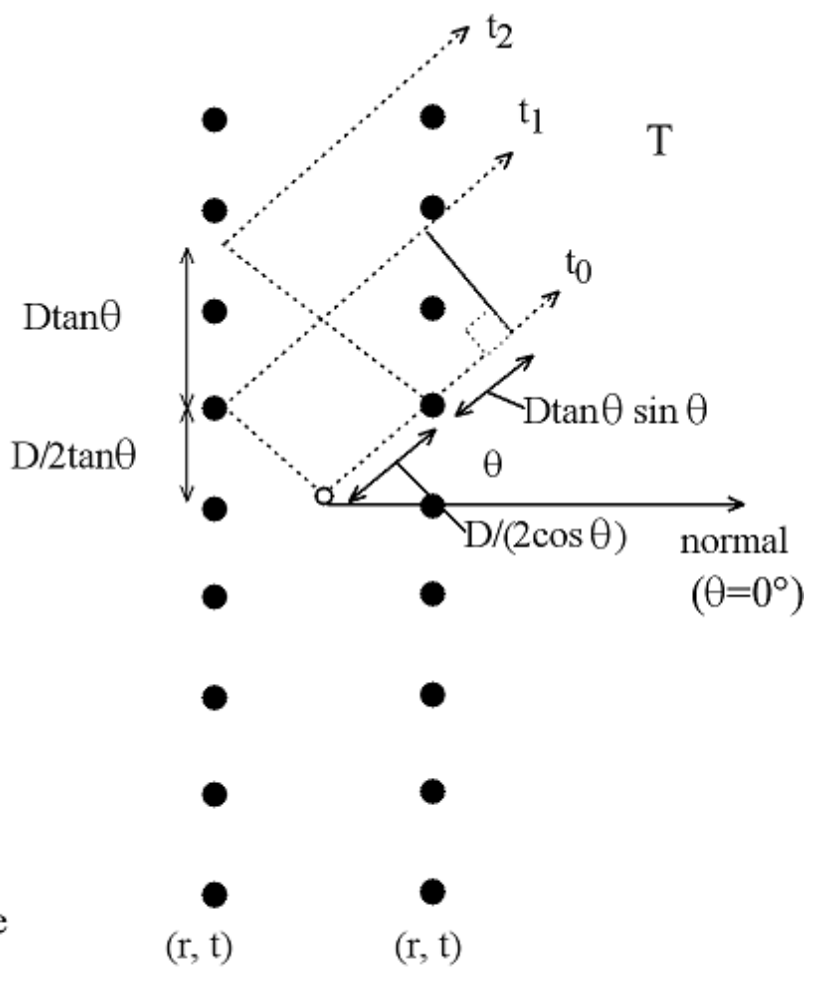

(b) 


\section{FIG.2}

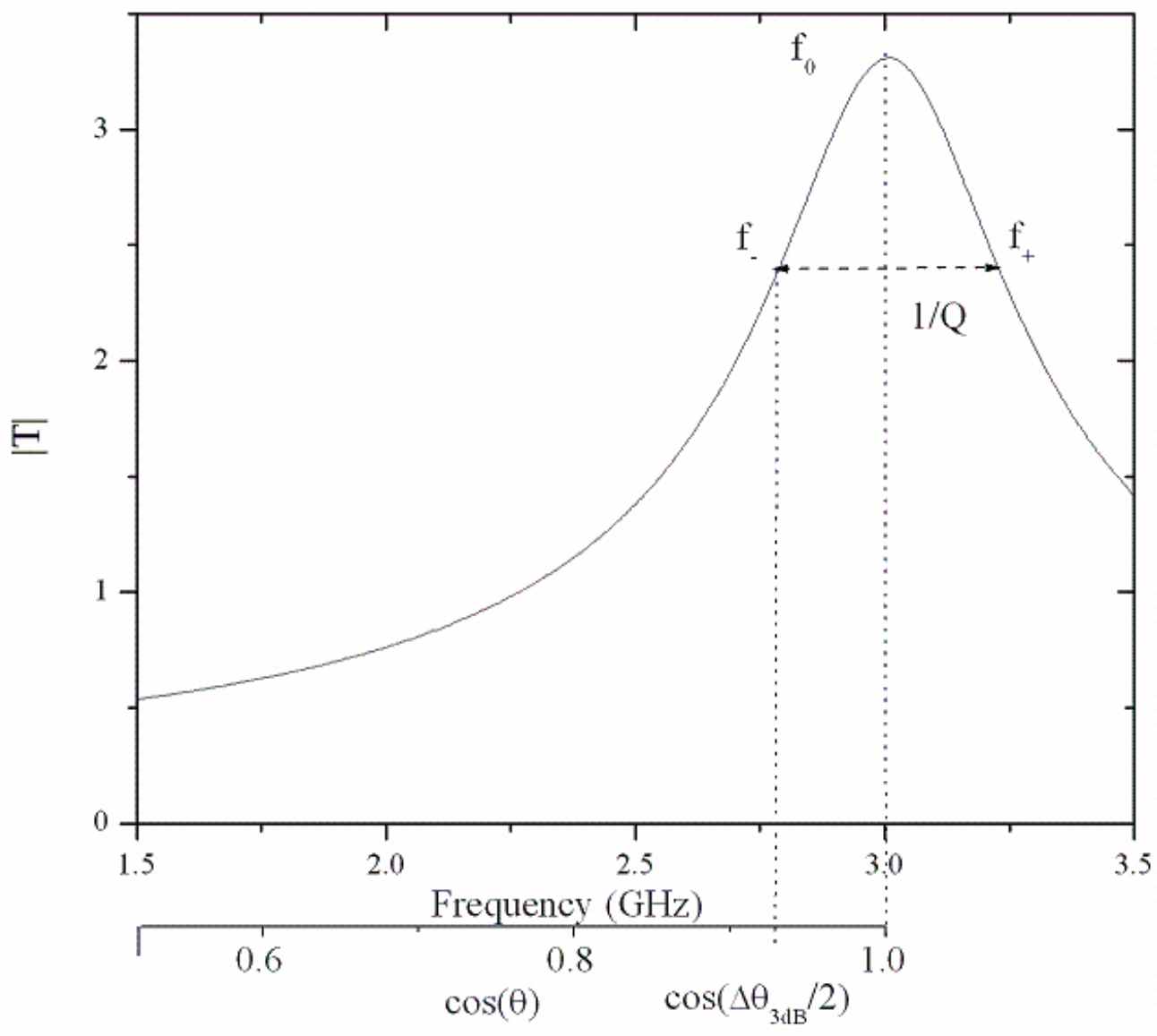




\section{FIG.3}

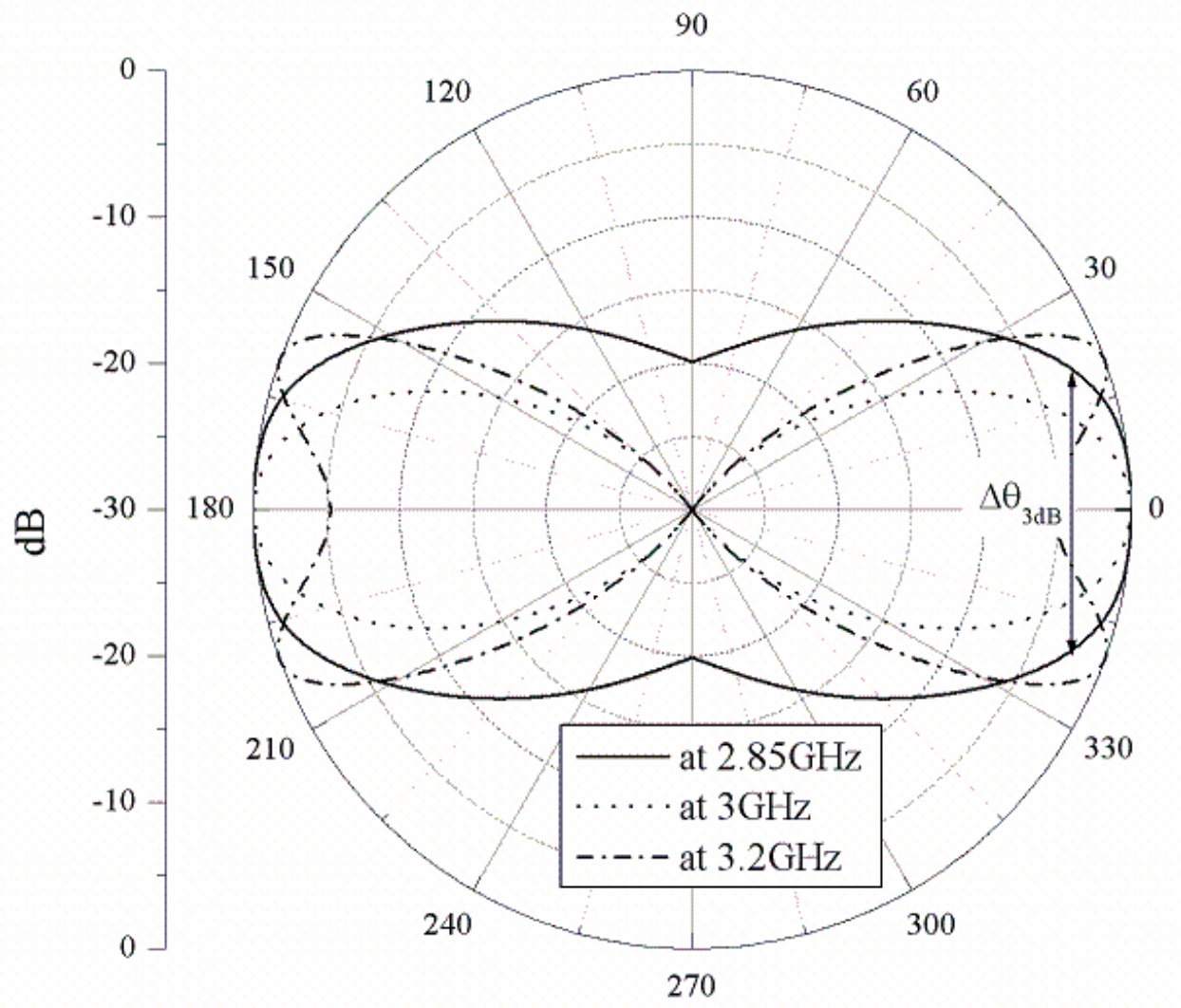


FIG. 4

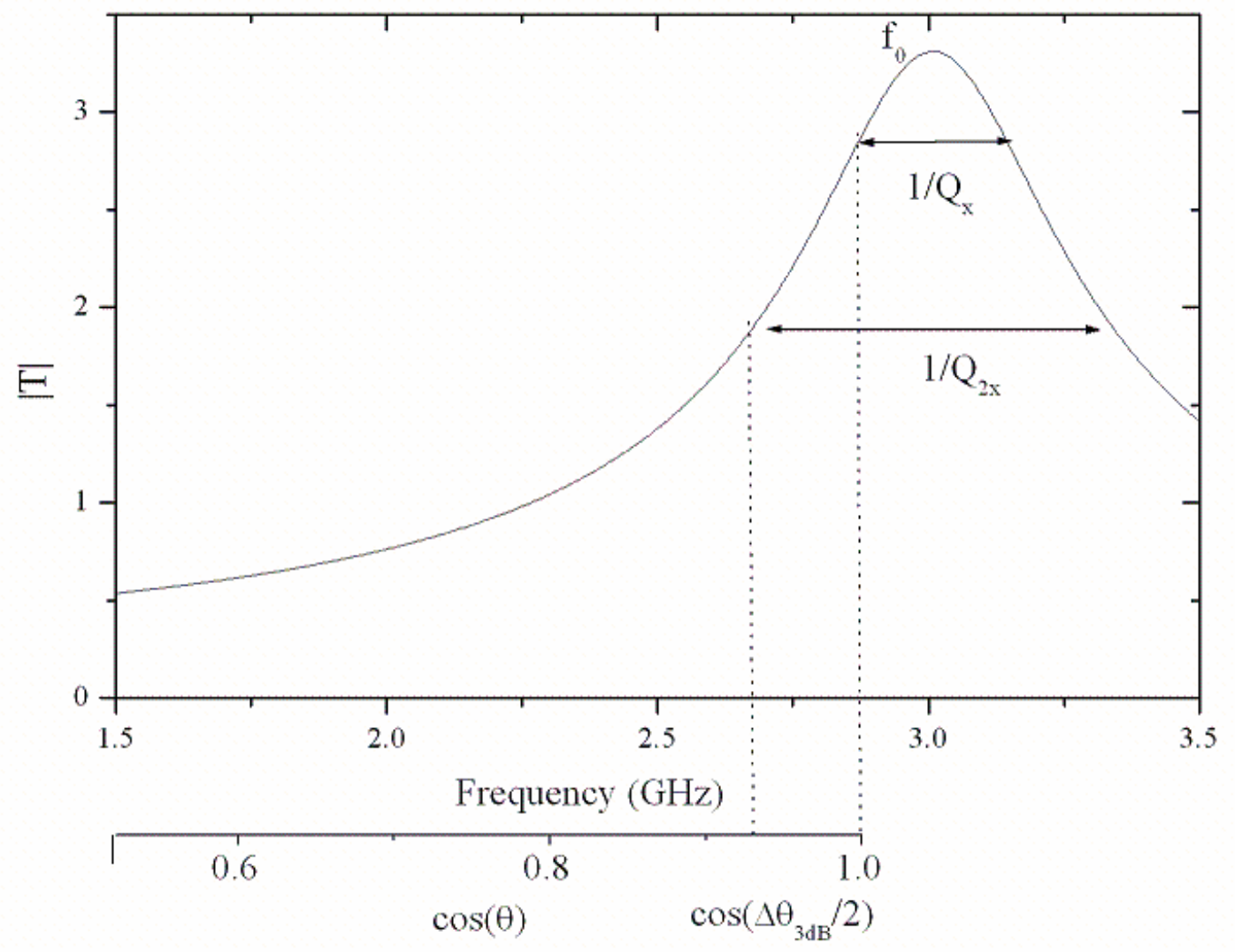




\section{FIG.5}

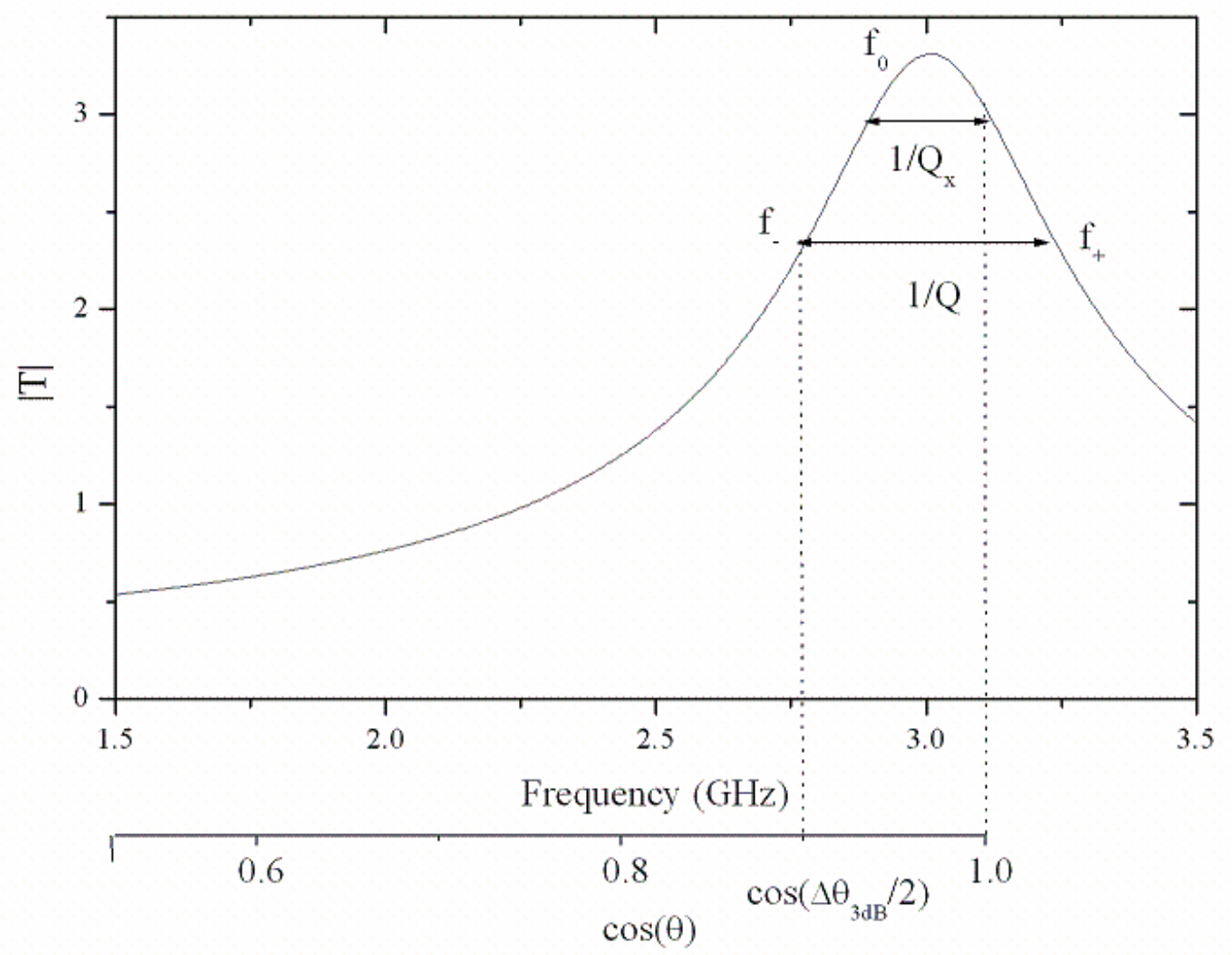


FIG.6

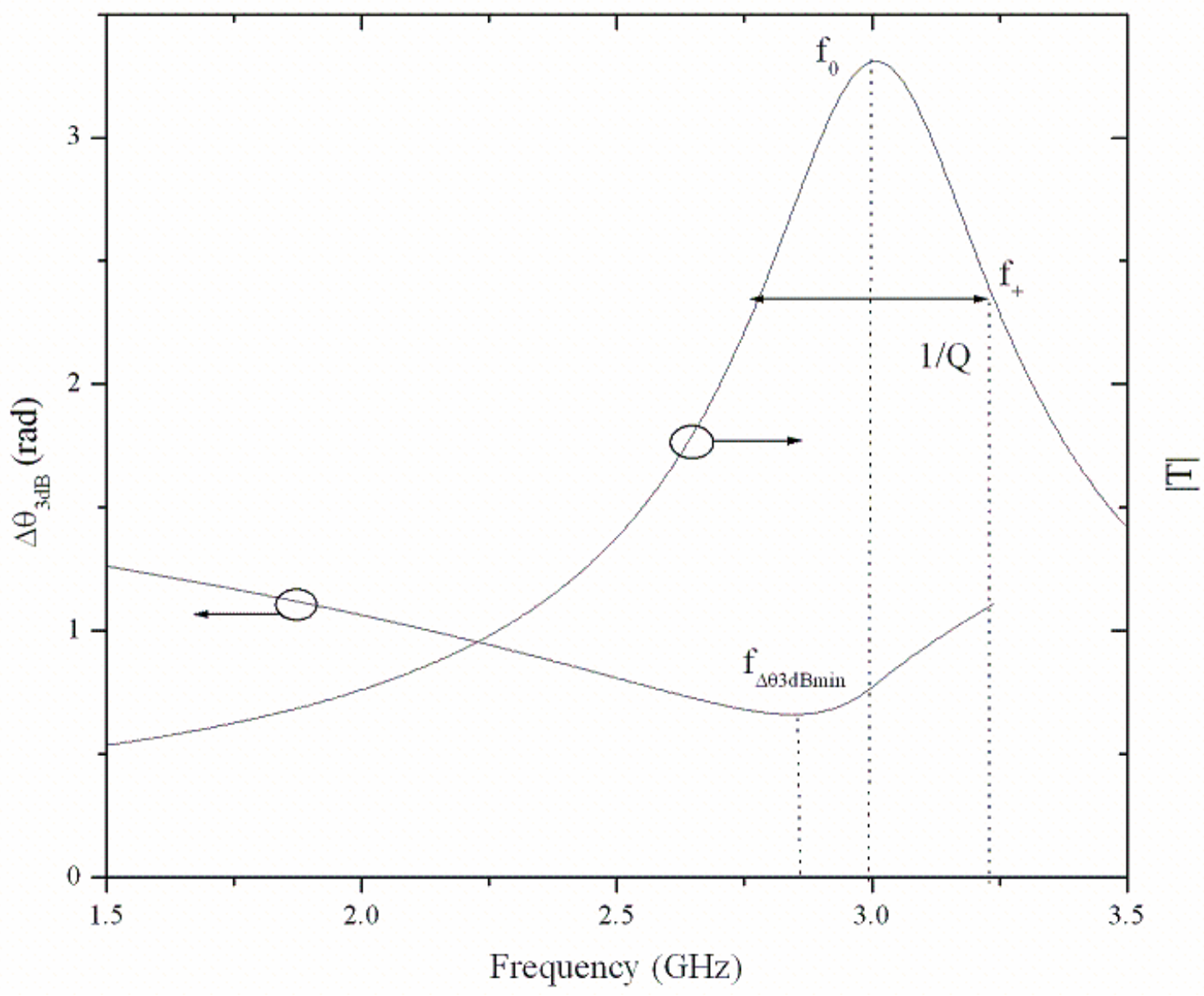


FIG. 7

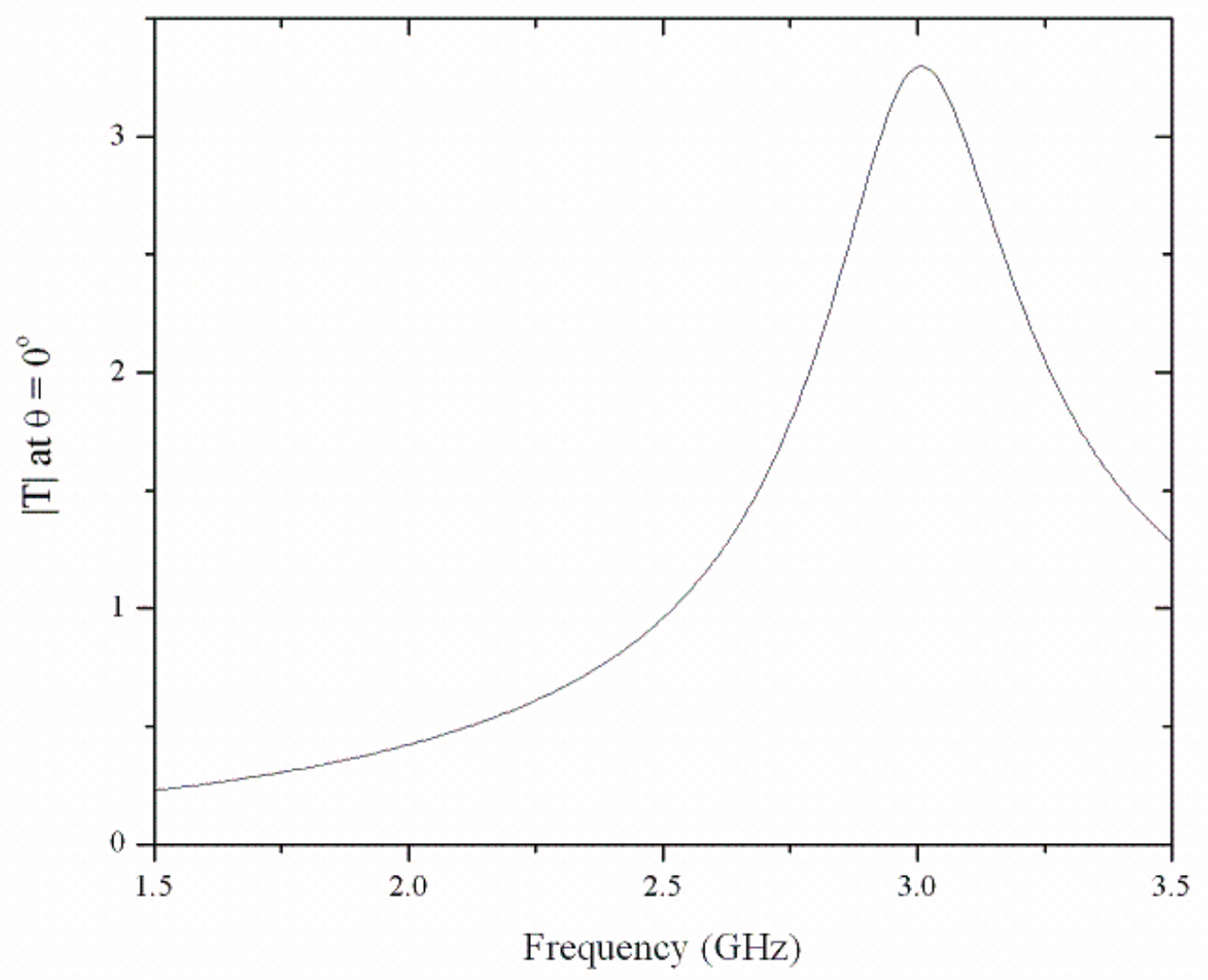


FIG.8

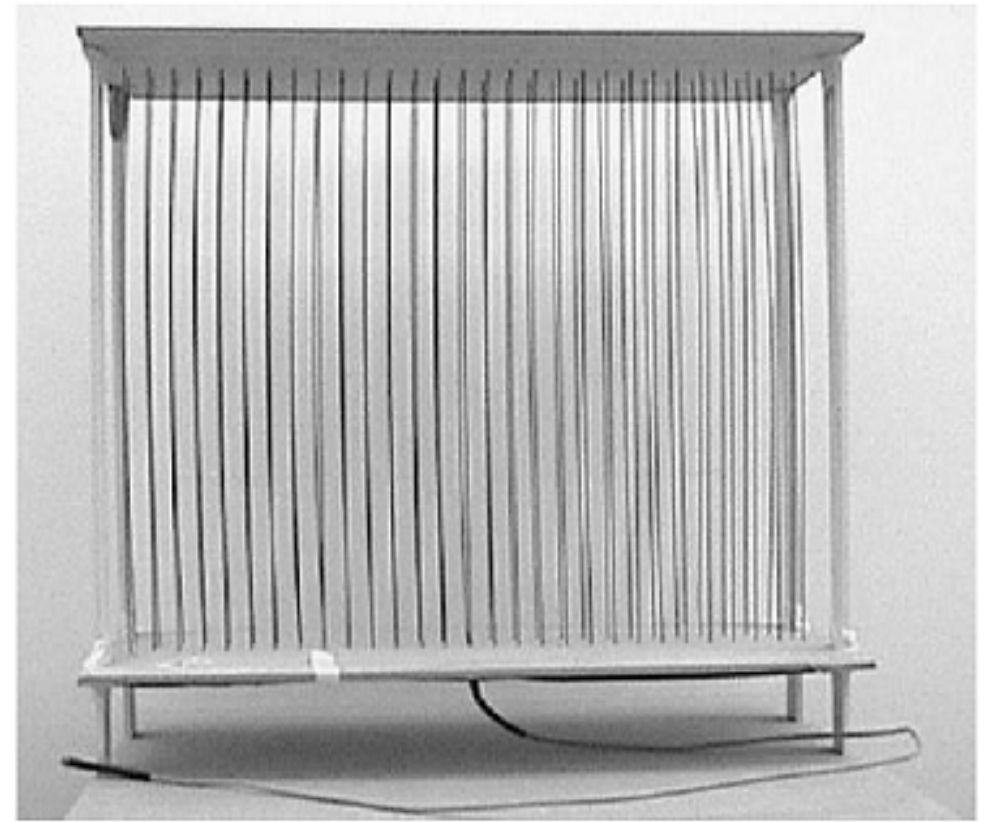




\section{FIG.9}

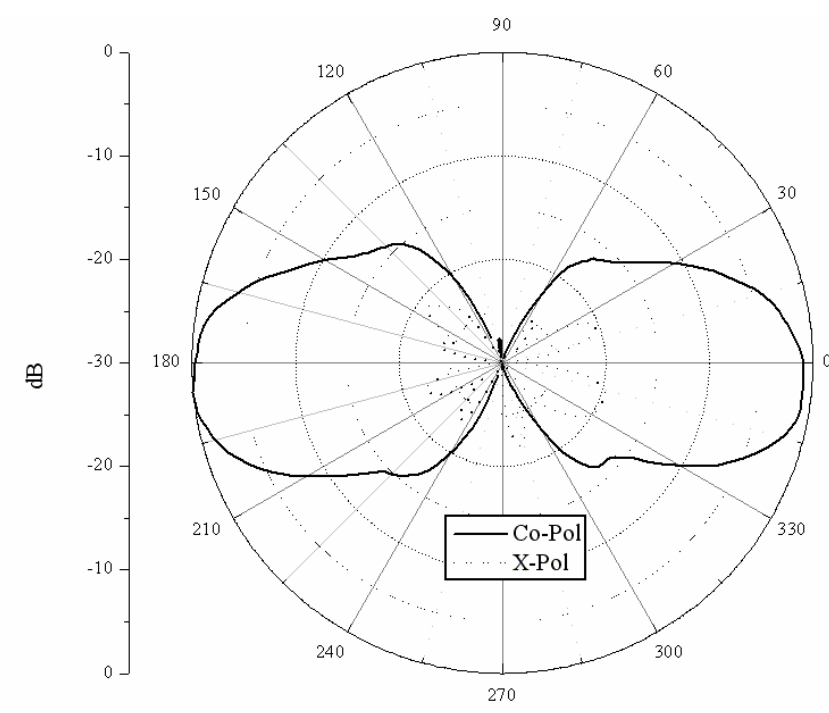

(a) H-plane

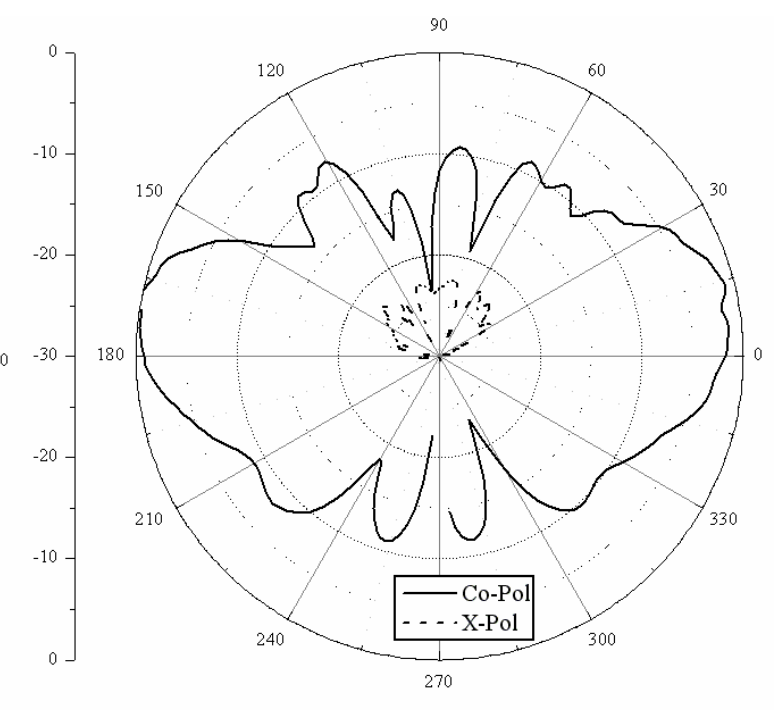

(b) E-plane 
FIG.10

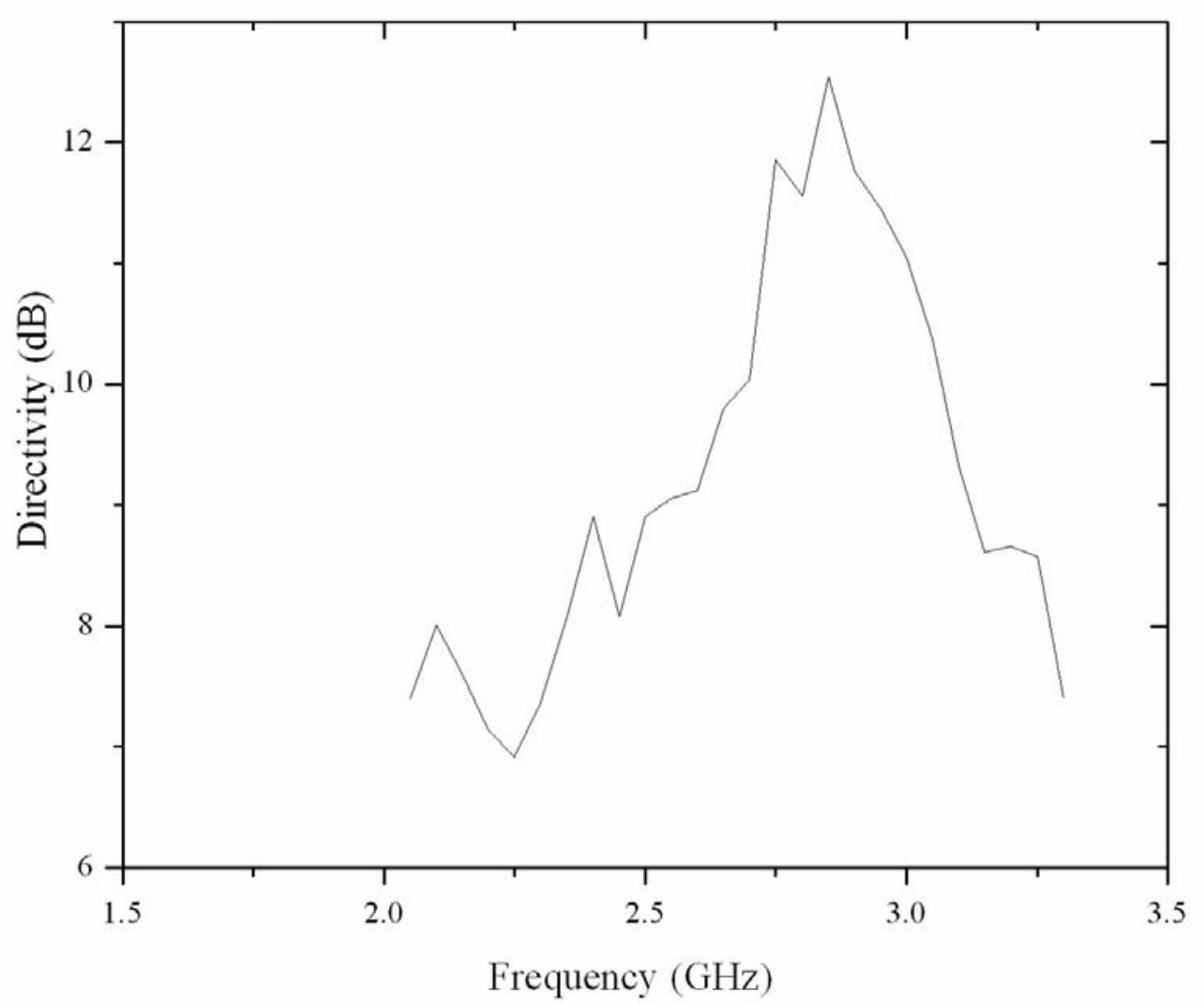

